International

Medical Society

http://imedicalsociety.org

\title{
Investigation of Oxidative Stress Status in Metabolic Syndrome Patients Using Lipid Peroxidation Biomarkers
}

\author{
Muhamed T Osman ${ }^{3}$, Rahman $\mathrm{T}^{1,2}$, Ismail TS. ${ }^{2}$, Azlina A.R. ${ }^{2}$, H. Nawawi ${ }^{1,2}$
}

\section{Abstract}

Metabolic syndrome (MS) is a cluster of interrelated common clinical disorders, including obesity, insulin resistance, glucose intolerance, hypertension and dyslipidaemia. Elevated oxidative stress has been suggested as the underlying causes in the development of MS. The aim of this study was to determine the relationship between oxidative stress and metabolic syndrome (MS) among Malaysians and to assess the oxidative stress status in the syndrome using lipid peroxidation biomarkers. This cross-sectional study involved a total of 260 subjects (Mean \pm SD: $53 \pm 11$ years, 66 Males) were randomly recruited and divided into five groups consisting of 52 patients for each group which are MS with newly diagnosed Diabetes Mellitus (MSDM), MS with Impaired Fasting Glucose (MSIFG), normoglycemia with MS (MSNG), central obesity without MS (OBXMS) and healthy subjects as a control group (NC). Serum samples were analysed for malondialdehyde (MDA) and oxidized low density lipoprotein (Ox LDL). Detailed history taking and physical examination is performed to obtain relevant clinical data. Fasting blood samples of all subjects were collected, separated, stored and biochemical analysis were done to determine the levels of oxidative stress biomarkers in serum. Comparison of MDA levels between MS patients and NC showed that among the other $3 \mathrm{MS}$ groups, MSDM had significantly higher level compared to NC $(p<0.0001)$ and OB XMS $(p<0.005)$. For Ox LDL, MSIFG had significantly higher level compared to NC and OBXMS $(p<0.0001)$. In conclusion, this study clearly indicates that oxidative stress is an associated contributor with metabolic syndrome by the evidence of increased level of lipid peroxidation biomarkers in serum
1 Institute of Pathology, Forensic and Laboratory Medicine (I-PPerForM), UiTM Malaysia.

2 Faculty of Medicine, Universiti Teknologi MARA (UiTM) Malaysia.

3 Faculty of Medicine and Defense Health, National Defense University of Malaysia (UPNM).

\section{Contact information:}

Hapizah Mohd. Nawawi. MD, MRCPath, FRCPath, FAMM.

Address: Institute of Pathology, Forensic and Laboratory Medicine (I-PPerForM) and Centre for Pathology Diagnostic and Research Laboratories, Faculty of Medicine, Universiti Teknologi MARA, Sg. Buloh Campus, Faculty of Medicine, Universiti Teknologi MARA, Jalan Hospital, 47000 Sg. Buloh, Selangor, Malaysia.

Tel: +603-61264616/4626, $+6012-3838075$

Fax: $+603-61264636$

झ hapizah.nawawi@gmail.com

Keywords

Metabolic Syndrome; Oxidative Test; Malondialdehyde; Oxidized Low Density Lipoprotein. 


\section{Introduction}

Metabolic syndrome (MS) is a cluster of interrelated common clinical disorders, including obesity, insulin resistance, glucose intolerance, hypertension and dyslipidemia [1]. This disorder was also known as syndrome $X$ and insulin-resistance syndrome [1]. The three most current definitions of metabolic syndrome, created by the National Cholesterol Education Program - Third Adult Treatment Panel (NCEP ATPIII), the World Health Organization (WHO), and the International Diabetes Foundation (IDF), provide useful guidelines to identify those individuals at increased risk for development of type 2 diabetes, atherosclerotic cardiovascular disease (CVD), and cardiovascular death [1-2]. The underlying mechanisms of the pathophysiology of MS presentations are common inflammatory stress conditions and an increase in the formation of free radicals (reactive oxygen and nitrogen species; RONS). It is the oxidative stress which may play a considerable role in the pathological development of diabetes and cardiovascular complications [3].

Oxidative stress (OX) is known to play a major role in the pathogenesis of MS [4]. Oxidative stress means an imbalance between production of reactive oxygen species and antioxidant defense system that buffers the oxidative damage [5], and is considered to be one of the main causes of molecular damage to cellular and tissue structures [6]. This imbalance in the rate at which the intracellular content of free radicals, produced through a number of cellular events, increases relative to the capacity of the cell to dispose of these oxidants [7], when not neutralized, these free radicals have the capacity to alter the integrity of numerous molecules such as lipids, proteins and DNA [7].

Lipids are a major target of free radical attack, which induces lipid peroxidation [8]. Free radicalinduced peroxidation of membrane lipids can be very damaging because it leads to alterations in the biophysical properties of the membrane, such as the degree of fluidity, and can lead to inactivation of membrane-bound receptors or enzymes, which in turn may impair normal cellular function [8-9]. Therefore, measurement of products of lipid peroxidation has been commonly used to assess oxidative stress/injury [8-9].

The most widely used test for oxidative stress is measurement of malondialdehyde (MDA), a product of lipid peroxidation, by a thiobarbituric acidreacting substances (TBARS) assay [10-11]. The excess MDA produced as a result of tissue injury have been implicated as causative agents in cytotoxic processes, and it is reasonable to suppose that releases from cell membranes may diffuse, interact, and induce oxidative modifications in other cells and thereby increasing the risk of cardiovascular damage in metabolic syndrome patients [10-11].

Presence of oxidized low-density lipoprotein (OxLDL) is another aspect of oxidative modification [12]. The concentration of circulating oxidized LDL (OxLDL) has shown relationships with atherosclerotic disease, and some studies indicate OxLDL concentrations to be increased in the metabolic syndrome. Although the origin of OxLDL is unknown, the vascular wall is considered the main source [12-13].

However, the oxidative stress status in patients with MS remains unclear. The challenge is to identify high risk individuals as through effective identification and intervention of risk factors, so that the disease can be halted successfully. One strategy is to understand the overlying pathogenesis and disease mechanisms which will possibly help identify surrogate markers to effectively evaluate the overall risk of these individuals. The aim of our study was to investigate status of oxidative stress in overall MS patients, MS patients with and without DM, Central obesity patients without MS, and lastly to determine the most reliable biomarker in patients with MS. 


\section{Materials and Methods}

Study design: Cross sectional study.

Study Group: Written informed consent and Ethical Committee approval was obtained prior to commencement of study. Metabolic Syndrome (MS) patients and controls were recruited from the Specialists Clinics of a Teaching Institution and from the health screening programmes. Diagnosis of MS was defined according to the International Diabetes Federation (IDF) criteria 2005 [14]. Healthy normal controls (normoglycaemia, normotensive, normal lipids) were also recruited in parallel. This cross-sectional study involved a total of 260 subjects who were divided into five groups consisting of $52 \mathrm{pa}$ tients for each group which were; (a) MS with newly diagnosed Diabetes Mellitus (MSDM); (b) MS with Impaired Fasting Glucose (MSIFG); (c) normoglycemia with MS (MSNG); (d) central obesity without MS (OBXMS) and (e) healthy subjects as a control group (NC). For each subject, a set of questionnaire was completed and detailed history taking and physical examination was performed to obtain the relevant clinical data. Blood samples were collected following 10-12 hours of fasting.

Subjects with MS were diagnosed according to the international IDF criteria, as follows: Central obesity is defined as waist circumference $\geq 90 \mathrm{~cm}$ for males and $\geq 80 \mathrm{~cm}$ for females plus at least 2 of the following four factors: Fasting triglycerides (TG) $\geq 1.7 \mathrm{mmol} / \mathrm{L}$; high density lipoprotein (HDL) for males $\geq 1.0 \mathrm{mmol} / \mathrm{L}$ and for females $\geq 1.3 \mathrm{mmol} / \mathrm{L}$; blood pressure $\geq 130 / 85 \mathrm{mmHg}$; and fasting glucose $(F G) \geq 6.1 \mathrm{mmol} / \mathrm{L}$. MSDM was defined as those with $F G \geq 7.0 \mathrm{mmol} / \mathrm{L}$. MSIFG was defined as plasma FG $=6.1-6.9 \mathrm{mmol} / \mathrm{L}$. MSNG was defined as $M S$ with $F G \geq 6.1 \mathrm{mmol} / \mathrm{L}$. OBXMS was defined as waist circumference of $\geq 90 \mathrm{~cm}$ for males and $\geq 80 \mathrm{~cm}$ for females and Body Mass Index (BMI) $\geq$ 25 with absence of MS. The exclusion criteria were current smoking, low density lipoprotein (LDL) $\geq$ $4.1 \mathrm{~mol} / \mathrm{L}$, on diabetic medication, patients on long term antioxidant therapy or those on anti-inflam- matory drugs (e.g. steroid, aspirin and NSAIDs), chronic inflammatory disorders or any condition limiting mobility, and severe diseases that shortens life expectancy.

Fasting blood samples of patients and controls were collected, separated, stored and analysed as appropriate. Blood were separated by centrifugation at 4500 rpm (Rotofix 32A, Hettich Zentrifugen, Germany) for 10 minutes. Plasma or serum were aliquoted into fresh $1.5 \mathrm{~mL}$ micro centrifuge tube (Camlab, England) and kept in $-80^{\circ} \mathrm{C}$ freezer until analysis. Serum samples were analyzed for several baseline biochemical tests-fasting serum lipids (FSL) and Fasting plasma glucose (FPG) by automated standard laboratory techniques (Cobas Integra 400plus, Roche Diagnostics, Switzerland).

\section{Biochemical Analysis}

Markers of oxidative stress that were measured; (1) Oxidative Low Density Lipoprotein (Ox LDL) level were measured by Enzyme Link Immunosorbent Assay (sandwich ELISA) using commercial ELISA immunoassay kit (Mercodia Uppsala, Sweeden) [15], and (2) Malondialdehyde (MDA) levels were analyzed using thiobarbituric acid (TBA) test [16]. This test was based on the measurement of pink chromogen products at the $532 \mathrm{~nm}$.

Ox LDL concentration in plasma was measured by a sandwich ELISA kit (Mercodia, Uppsala, Sweden) utilizing the same specific plate coated with murine monoclonal antibody, mAb-4E6 [15]. Using this antibody (mAb-4E6) it is possible to measure very small amounts Ox LDL containing a conformational epitope in the apoB-100 moiety of LDL that is generated as a consequence of substitution of lysine residues of apoB-100 with aldehydes. The specificity for the antibody mAb-4E6 has been assessed, showing that $50 \%$ inhibition of binding of mAb-4E6 to immobilized Ox LDL was obtained with 0.025 mg dL-1 Cu2+ OxLDL and with $25 \mathrm{mg} \mathrm{dL}-1$ native LDL.

6 vials of standards $(0,1.3,2.8,5.1,10$, and $20 \mathrm{mU} / \mathrm{l}), 1$ vial assay buffer, 1 vial anti-apoB, 1 vial 
conjugate stock solution, 1 vial conjugate buffer, anti-oxidized LDL 12x8 wells coated microtitration strips (breakable strips), 1 vial peroxidase substrate, 1 vial stop solution, 1 bottle wash buffer, 1 bottle sample buffer, and 2 vials controls $\mathrm{L}$ and $\mathrm{H}$.

Malondialdehyde (MDA): MDA was measured by the method adapted from Ledwozyw et al., (1986) [16]. This test was based on the measurement of pink chromogen products at the $532 \mathrm{~nm}$. This product is a result of the reactions between TBA and MDA, the last product of fatty acid peroxidation. Sodium hydroxide, 1,1,3,3-tetraetoxypropane (TEP), n-butanol and acid Trichloroacetic (TCA) were obtained from Sigma - Aldrich, USA. Hydrochloric acid $(\mathrm{HCl})$, Thiobarbituric acid (TBA), Sodium Chloride, Potassium dihydrogen phosphate and dipotassium hydrogen phosphate were purchased from Merck, Germany.

\section{Statistical Analysis}

Data were expressed as mean \pm SD or median $(50 \%$ $\mathrm{Cl}$ ) depending results from normality testing determine by Kolmogorov Smirnov test. For between group differences, Mann-Whitney test was used. For comparison between study groups, Kruskal Wallis tests were performed. Correlation between two continuous data was analyzed by Spearman correlation coefficient tests. The data was calculated with statistical analysis system software, SPSS for Windows version 14.0 (Chicago, IL, USA).

\section{Results}

Table 1 showed the demographic data of the overall recruited subjects. Subjects were matched for gender and smoking status ( $p=0.987$ and $p=0.069$, respectively). All MS subjects had lower high density lipoprotein cholesterol (HDL-c; $p<0.0001)$, higher LDL-C, and higher TC and triglycerides (TG; $\mathrm{p}<0.0001$ ) compared to NC. However, HDL- $c(p<0.198)$ and gender $(p<0.645)$ were matched using the multi linear regression analysis.

\section{Malondialdehyde (MDA) and Oxidative Low Density Lipoprotein (0x LDL) levels in subjects. All $156 \mathrm{MS}$ patients with 52 obesity without MS (OBXMS) and}

Table 1. Baseline characteristics of MS patients and Normal Control subjects for the cross sectional study.

\begin{tabular}{|c|c|c|c|c|c|c|c|}
\hline \multirow{2}{*}{ Parameters } & MSNG & MSIFG & MSDM & All MS & OBXMS & Normal & \multirow{2}{*}{$p$} \\
\hline & $N=52$ & $N=52$ & $N=52$ & $N=156$ & $N=52$ & $\mathrm{~N}=52$ & \\
\hline Gender (M/F) & $1.7 \pm 0.4$ & $1.6 \pm 0.5$ & $1.8 \pm 0.4$ & $1.7 \pm 0.5$ & $1.6 \pm 0.5$ & $1.6 \pm 0.5$ & $* * *$ \\
\hline Age (Years) & $52.8 \pm 9.9$ & $57.7 \pm 10.5$ & $52.7 \pm 8.8$ & $54.4 \pm 10.0$ & $51.9 \pm 11.3$ & $48.3 \pm 10.7$ & NS \\
\hline$W C(\mathrm{~cm})$ & $90.0 \pm 9.8$ & $90.4 \pm 7.7$ & $94.7 \pm 9.7$ & $91.7 \pm 7.9$ & $92.6 \pm 14.5$ & $73.9 \pm 8.7$ & NS \\
\hline $\mathrm{SBP}(\mathrm{mm} \mathrm{Hg})$ & $144.1 \pm 24.9$ & $149.4 \pm 24.4$ & $153.6 \pm 23.4$ & $149.0 \pm 24.4$ & $138.8 \pm 22.7$ & $115.6 \pm 12.4$ & NS \\
\hline $\mathrm{DBP}(\mathrm{mm} \mathrm{Hg})$ & $85.8 \pm 13.5$ & $85.0 \pm 12.0$ & $91.2 \pm 13.8$ & $87.3 \pm 13.3$ & $82.6 \pm 12.3$ & $69.6 \pm 7.8$ & NS \\
\hline $\mathrm{HDL}-\mathrm{c}(\mathrm{mmol} / \mathrm{L})$ & $1.1 \pm 0.2$ & $1.2 \pm 0.3$ & $1.3 \pm 0.2$ & $1.4 \pm 0.3$ & $1.4 \pm 0.2$ & $1.5 \pm 0.3$ & $* * *$ \\
\hline LDL-c (mmol/L) & $3.7 \pm 0.9$ & $4.1 \pm 1.2$ & $3.8 \pm 1.0$ & $3.8 \pm 1.0$ & $3.8 \pm 1.1$ & $3.4 \pm 0.8$ & NS \\
\hline $\mathrm{TC}(\mathrm{mmol} / \mathrm{L})$ & $5.8 \pm 1.0$ & $6.3 \pm 1.3$ & $6.3 \pm 1.1$ & $6.1 \pm 1.1$ & $5.8 \pm 1.2$ & $5.4 \pm 0.9$ & NS \\
\hline $\mathrm{TG}(\mathrm{mmol} / \mathrm{L})$ & $2.3 \pm 0.8$ & $2.2 \pm 0.9$ & $2.7 \pm 1.4$ & $2.4 \pm 1.1$ & $1.3 \pm 0.4$ & $1.2 \pm 0.5$ & $* * *$ \\
\hline
\end{tabular}

Data are expressed as Mean \pm SD. ${ }^{* * *} p=<0.0001 ;{ }^{* *} p=<0.001 ;{ }^{*} p<0.05 ; N S=$ Not Significant M/F indicates male/female; WC, Waist Circumference; SBP, Systolic Blood Pressure; DBP, Diastolic Blood Pressure; HDL-C, High Density Lipoprotein Cholesterol; LDL-C, Low Density Lipoprotein Cholesterol; TC, Total Cholesterol; TG, Triglycerides. MSNG, metabolic syndrome with normoglycemia; OBXMS, obesity without metabolic syndrome; and NC is normal control. 


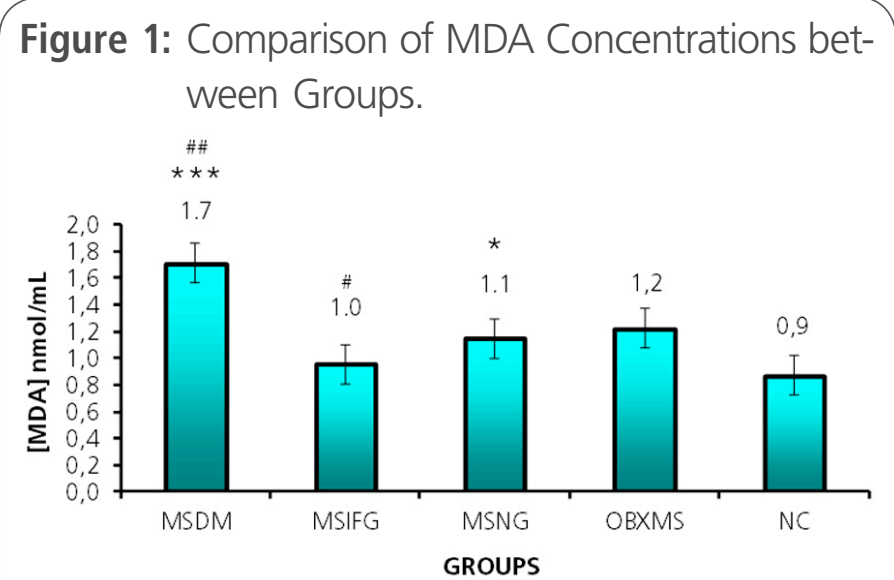

Comparison of MDA levels between MS patients and NC. Among the other 3 MS groups, MSDM had significantly higher level compared to NC; $p<0.0001$ and OB XMS; $p<0.005$. Data are expressed as median.

${ }^{* * *} p<0.0001,{ }^{* *} p<0.005,{ }^{*} p<0.05$ compared to NC. \#\#\# $p<0.0001, \# \# p<0.005$, \# $p<0.05$ compared to OB XMS.

52 NC subjects were analyzed for their oxidative stress biomarkers. Comparison of the above biomarkers between all patients group are shown in Figure 1 and Figure 2. For MDA, among the other 3 MS groups, MSDM had significantly higher level compared to NC; $p<0.0001$ and compared to OB $X M S ; p<0.005$. MSIFG shows slightly significant increased compared to NC; $p<0.05$ and for MSNG, the MDA levels also had slightly significant increased compared to NC; $p<0.05$. As for the Ox LDL levels, MSIFG had significantly higher level when compared to $N C$ and compared to OB XMS; $p<0.0001$. MSDM also have an increased level when compared to NC $(p<0.005)$.

Association between Metabolic Syndrome (MS), Obesity without MS (OBXMS), and Normal Control (NC) group with Malondialdehyde (MDA) and Oxidative Low Density Lipoprotein (0x LDL) levels. All 156 MS patients with 52 obesity with no MS (OBXMS) and 52 NC subjects were analyzed for the association between patients group and the oxidative stress biomarkers. Comparison of the above biomarkers between all patients group are shown in Table 2 and Table 3. For MDA, 73 (46.79\%) patients over 156 patients have higher MDA levels for the all MS groups while OBXMS group have 25 (16.02\%)

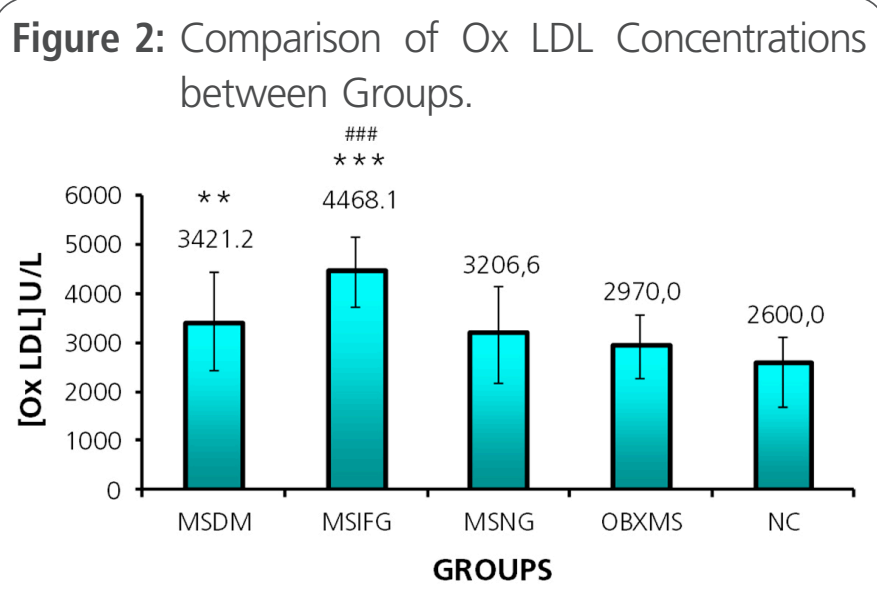

Comparison of Ox LDL levels between MS patients and NC. Among the other 3 MS groups, MSIFG had significantly higher level compared to NC and OB XMS; $p<0.0001$. Data are expressed as median $[50 \% \mathrm{Cl}]$.

${ }^{* *} p<0.0001,{ }^{* *} p<0.005,{ }^{*} p<0.05$ compared to NC. $\# \# \#<0.0001, \# \# p<0.005, \# p<0.05$ compared to OB XMS.

Table 2. Association between Metabolic Syndrome (MS), Obesity without MS, and Normal Control group with Malondialdehyde (MDA) Biomarker.

\begin{tabular}{|c|c|c|c|}
\hline \multirow{2}{*}{ Groups } & \multicolumn{2}{|c|}{ MDA } & \multirow{2}{*}{ Total } \\
\hline & NORMAL (-) & HIGH (+) & \\
\hline All MS & 83 & 73 (46.79\%) & 156 \\
\hline OBXMS & 27 & $25(16.02 \%)$ & 52 \\
\hline Normal & 37 & 15 (9.6\%) & 52 \\
\hline \multirow[t]{2}{*}{ Total } & 147 & 113 & 260 \\
\hline & \multicolumn{3}{|c|}{$\begin{array}{l}\text { This test are using chi square test } \\
\qquad p \text { value for MDA is } p<0.059\end{array}$} \\
\hline
\end{tabular}

Table 3. Association between Metabolic Syndrome (MS), Obesity without MS, and Normal Control group with Oxidative Low Density Lipoprotein (Ox LDL) Biomarker.

\begin{tabular}{|c|c|c|c|}
\hline \multirow{2}{*}{ Groups } & \multicolumn{2}{|c|}{ Ox LDL } & \multirow{2}{*}{ Total } \\
\hline & NORMAL (-) & $\mathrm{HIGH}_{(+)}$ & \\
\hline MS & 152 & $4(2.56 \%)$ & 156 \\
\hline OBXMS & 52 & $0(0 \%)$ & 52 \\
\hline Normal & 52 & $0(0 \%)$ & 52 \\
\hline \multirow[t]{2}{*}{ Total } & 256 & 4 & 260 \\
\hline & \multicolumn{3}{|c|}{$\begin{array}{l}\text { This test are using chi square test. } \\
\qquad p \text { value for MDA is } p<0.059\end{array}$} \\
\hline
\end{tabular}


patients over 27 (17.3\%) patients that have higher MDA levels. Normal control group only have 15 (9.6\%) subjects that have higher MDA levels over 37 subjects. This association is proven using the chi square test with $p<0.059$. The MDA results differ greatly with the Ox LDL result where the MS groups only have 4 (2.56\%) patients that have higher level of Ox LDL compared with lower level MDA patients in that groups. For the OBXMS and NC groups, none of the subjects have higher Ox LDL level $(p<$ 0.258).

All MS indicates the $3 \mathrm{MS}$ group which is MSDM, metabolic syndrome with diabetes mellitus; MSIFG, metabolic syndrome with impaired fasting glucose; MSNG, metabolic syndrome with normoglycemia; OBXMS, obesity without metabolic syndrome; and $\mathrm{NC}$ is normal control.

\section{Discussion}

Increased oxidative stress and inflammatory biomarkers are known to play an important role in the initiation and progression of atherosclerotic vascular disease [17-18], a major cause of morbidity and mortality in the world. The presence of vascular risk factors led to the identification of a unique pathophysiological condition called metabolic syndrome [1], now known to be characterized by elevated inflammatory markers and increased oxidative stress that can predict cardiovascular events such as the risk of myocardial infarction, stroke, and peripheral arterial disease [19-20]. Importantly, the risk for Coronary Heart Disease (CHD) is markedly greater in obese individuals with MS compared with those without it [21-22]. However, the association and effect of MS on plasma biomarkers of oxidative stress is largely unknown. In this study we tested our hypothesis that markers of oxidative stress can differentiate between individuals with or without MS. We also studied the individual ability of these biomarkers in predicting MS in an otherwise healthy person. To date, there are conflicting data about the associations among metabolic syndrome, inflammation, oxidative stress and lipids biomarkers [23-24].

Cross-sectional data from the present well characterized cohort of $>40$ years old subjects according to International Diabetes Federation (IDF) 2005 [14], criteria identified 156 from 260 subjects have MS. As depicted in table 1, group comparisons revealed differences for established features of MS with MS subjects have larger waist circumference (WC), lower High Density Lipoprotein cholesterol (HDL-C), higher Blood Pressure (BP), Total Cholesterol (TC), Triglycerides (TG) and Low Density Lipoprotein cholesterol (LDL-C) compared to those without MS.

When central obesity coupled with more than one criterion [14] are present, a diagnosis of MS can be made. Central obesity, recognized by increased WC, is the first criterion listed. Its inclusion reflects the priority given to central obesity as a contributor to MS. It has been established that abdominal obesity, assessed by WC, explained obesity-related health risk significantly better than body mass index (BMI) when MS was taken as an outcome measure [25]. Also listed are raised TG, reduced HDL-C, elevated BP, and raised TC and LDL-C. Results of the prospective Québec Cardiovascular Study have revealed that the presence of some features of MS found in viscerally obese men was predictive of a substantially increased risk of coronary heart disease (CHD) [26].

Hypertriglyceridaemia is associated with several atherogenic factors including increased concentrations of triglyceride-rich lipoproteins and the atherogenic lipoprotein phenotype consisting of high LDL-C and low HDL-c. The presence of elevated serum TG substantially increases the risk of CVD. Increased levels of LDL are one of the underlying symptoms that will trigger a diagnosis of MS and fasting hypertriglyceridaemia has been reported to be the best predictor of the presence of LDL particles. HDL is an important fat carrier responsible 
for transporting excess fat from cells back to the liver. Low level of HDL concentration is a common feature encountered in MS. According to different literatures, $65-83 \%$ of patients with the MS had low HDL-c. About $10-15 \%$ of healthy subjects had $\mathrm{HDL}-\mathrm{c}$ levels less than the $15^{\text {th }}$ percentile for age and gender. The relationship between the low HDL$\mathrm{C}$ and coronary artery disease has been shown in prospective epidemiological studies. A decreased plasma level of HDL-c is a major risk factor for coronary artery disease, which is the major cause of death in North American Society [27].

The results obtained in our study showed that systemic oxidative stress is significantly elevated in MS. The mechanisms involved in elevation of oxidative stress may include increased production of superoxide anion via the nicotinamide adenosine diphosphate oxidase pathway observed during hypertriacylglyceridemia, hypertension and obesity [28]. The occurrence of small dense LDL particles, which are more susceptible to oxidation than large buoyant LDL [29] is proportional to the degree of hypertriacylglyceridemia. A low HDL-C level contributes to oxidative stress, apart from other mechanisms, due to diminished antioxidative capacity [30].

In Figure 1, MDA level was significantly increased in all the MS groups compared to NC with MSDM shows higher MDA level among the group tested $(p<0.0001)$. Oxidative stress plays critical roles in the pathogenesis of various diseases. In the diabetic condition, oxidative stress impairs glucose uptake in muscle and fat and decreases insulin secretion from pancreatic $\beta$ cells. Increased oxidative stress also underlies the pathophysiology of hypertension and atherosclerosis by directly affecting vascular wall cells. These data are in a good agreement with other studies, [10, 31], that conclude increased oxidative stress in accumulated fat is, at least in part, the underlying cause of the development of metabolic syndrome [10, 31]. MDA have been implicated as causative agents in cytotoxic processes, and it is reasonable to suppose that releases from cell membranes may diffuse, interact, and induce oxidative modifications in other cells and in LDL molecules, thereby increasing the risk of CVD [10].

The Ox LDL level was demonstrated in figure 2 . Among the other 3 MS groups, MSIFG had significantly higher level compared to NC and compared to OB XMS; $p<0.0001$. These results are in line with other study [32] that showed association between the metabolic syndrome and circulating oxidized LDL in the Health, Aging, and Body Composition (Health $A B C$ ) cohort. It was observed in study that the MS was associated with higher concentrations of Ox LDL, because of a higher fraction of Ox LDL, and not to higher concentrations of LDL cholesterol. The odds of persons with the MS having high Ox $\mathrm{LDL}(>1.90 \mathrm{mg} / \mathrm{dL})$ concentrations was twice that of those not having the metabolic syndrome [32]. In the present study, Ox LDL was captured with the monoclonal antibody mAb-4E6, developed by Holvoet et al. [32], recognizing a conformational epitope in apoB-100 within LDL, which contains aldehyde-substituted lysine residues. Several clinical studies have shown circulating Ox LDL measured with mAb-4E6 to be associated with atherosclerotic disease. These Ox LDL particles are considered minimally modified LDL of unknown origin. Plasma levels of Ox LDL are influenced mainly by the degree of local oxidative stress in the arterial wall and susceptibility of LDL to oxidation. Ox LDL induces transformation of macrophages into foam cells that occur in the intima of the artery wall thus giving raise to other cardiovascular diseases such as atherosclerosis which is one of the risk factors in MS $[13,33]$.

\section{Conclusions}

In conclusion, this study clearly indicates that oxidative stress is associated with metabolic syndrome by the evidence of increased level of lipid peroxidation biomarkers in human serum. Further, oxLDL, MDA 
can be utilized to identify sunjects with MS (1) we identified the OXLDL as the best predictive biomarkers for MS in Malaysian population in comparison to other biomarkers. Further research will validate its use to diagnose people with MS compared to internationally defined criteria to ultimately yield a cost-effective method for MS diagnosis and CAD risk stratification. Moreover, further studies are needed to show the association between oxidative stress markers, DM, cardiovascular diseases and MS. The correlation between duration of MS, severity of metabolic disorders in MS, oxidative stress and cardiovascular risk are needed to be shown in further studies.

\section{Acknowledgement}

The authors would like to express appreciation to the Ministry of Higher Education, Malaysia for financial support under the Fundamental Research Grant Scheme (FRGS) code 600-IRDC/ST/FRGS.5/3/1199 for the grant awarded to corresponding author, Universiti Teknologi MARA for the laboratory facilities and Siti Nurul Azwa Yusof from Management Science University, Shah Alam, Selangor, Malaysia for her technical assistance.

\section{Competing interests}

The authors declare that they have no conflict interest.

\section{References}

1. Moller DE and Kaufman KD. Metabolic syndrome: A clinical and molecular perspective. Annu. Rev. Med 2005; 56: 45-62.

2. Alberti G, Zimmet P, Shaw J, Grundy SM. The IDF consensus worldwide definition of the metabolic syndrome. International Diabetes Foundation 2006; 1: 23.

3. Jiri Skalicky, Vladimira Muzakova, Roman Kandar, Milan Meloun, Tomas Rousar and Vladimir Palicka. Evaluation of oxidative stress and inflammation in obese adults with metabolic syndrome. Clin Chem Lab Med 2008; 46(4): 499-505
4. Mustafa zbek, Evrim akır, Enis zkaya. Oxidative Stress Markers in Metabolic Syndrome. Turk Jem 2011; 15: 57-61

5. Betteridge DJ. What is the oxidative stress? Metabolism 2000; 4913-18.

6. Holvoet P, Lee D.H, Steffes M, Gross M, Jacobs DR Jr. Association between circulating oxidized low-density lipoprotein and incidence of the metabolic syndrome. JAMA 2008; 299: 228793.

7. Goldstein, B. J. and R. Scalia. Adiponectin: A Novel Adipokine Linking Adipocytes and Vascular Function. J Clin Endocrinol Metab 2004; 89(6): 2563-2568.

8. Montuschi, P., P. J. Barnes. Isoprostanes: markers and mediators of oxidative stress." FASEB J 2004; 18(15): 1791-1800.

9. Usta M, Turan E, Aral H, Inal BB, Gurel MS, Guvenen G. Serum paraoxonase-1 activities and oxidative status in patients with plaquetype psoriasis with/without metabolic syndrome. J Clin Lab Anal 2011; 25: 289-95.

10. Redon, J., M. R. Oliva. Antioxidant Activities and Oxidative Stress Byproducts in Human Hypertension. Hypertension 2003; 41(5): 1096-1101.

11. Kand'a' r R, Z` a' kova' $P$, Muža'kova' V. Monitoring of antioxidant properties of uric acid in humans for a consideration measuring of levels of allantoin in plasma by liquid chromatography. Clin Chim Acta 2006; 365: 249-56.

12. Sjogren, P., S. Basu. "Measures of Oxidized Low-Density Lipoprotein and Oxidative Stress Are Not Related and Not Elevated in Otherwise Healthy Men With the Metabolic Syndrome." Arterioscler Thromb Vasc Biol 2005; 25(12): 25802586.

13. Wallenfeldt, K., B. Fagerberg. Oxidized low-density lipoprotein in plasma is a prognostic marker of subclinical atherosclerosis development in clinically healthy men. Journal of Internal Medicine 2004; 256(5): 413-420.

14. Zimmet, P., K. Alberti. A New International Diabetes Federation (IDF) Worldwide Definition of the Metabolic Syndrome: the Rationale and the Results." Revista Española de Cardiología (Internet) 2005; 58(12): 1371-1375.

15. Holvoet, P., J. Vanhaecke. Oxidized LDL and MalondialdehydeModified LDL in Patients With Acute Coronary Syndromes and Stable Coronary Artery Disease. Circulation 1998; 98(15): 14871494.

16. Ledwozyw, A., Michalak, J., Stepien A. and Kadziolka, A. The relationship between plasma triglycerides, cholesterol, total lipids and lipid peroxidation products during human atherosclerosis. Clin. Chim. Acta 1986; 155: 275-284.

17. S. I. Toshima, A. Hasegawa,M. Kurabayashi. Circulating oxidized low density lipoprotein levels: a biochemical risk marker for coronary heart disease, Arteriosclerosis, Thrombosis, and Vascular Biology 2000; 20: 2243-2247. 
18. P. Libby, P. M. Ridker, and A. Maseri. Inflammation and atherosclerosis, Circulation 2002; 105: 1135-1143.

19. B. Hansel, P. Giral, E. Nobecourt. Metabolic syndrome is associated with elevated oxidative stress and dysfunctional dense high-density lipoprotein particles displaying impaired antioxidative activity. Journal of Clinical Endocrinology and Metabolism 2004; 89: 4963-4971.

20. M. Jacobs, M. M. J. Van Greevenbroek, C. J. H. Van Der Kallen. Low-grade inflammation can partly explain the association between the metabolic syndrome and either coronary artery disease or severity of peripheral arterial disease: the CODAM study. European Journal of Clinical Investigation 2009; 39-6: 437-444.

21. J.-P. Despres. Inflammation and cardiovascular disease: is abdominal obesity the missing link?. International Journal of Obesity 2003; 27-3: S22-S24.

22. H. M. Lakka, D. E. Laaksonen, T. A. Lakka. The metabolic syndrome and total and cardiovascular disease mortality in middle-aged men. Journal of the American Medical Association 2002; 288- 21: 2709-2716.

23. Valle Gottlieb MG, da Cruz IB, Duarte MM. Associations among Metabolic Syndrome, Ischemia, Inflammatory, Oxidatives, and Lipids Biomarkers. J Clin Endocrinol Metab 2010; 95: 586-91.

24. Duarte MM, Rocha JB, Moresco RN. Association between ischemia-modified albumin, lipids and inflammation biomarkers in patients with hypercholesterolemia. Clin Biochem 2009; 42: 666-71

25. Janssen, I., P. T. Katzmarzyk. Waist circumference and not body mass index explains obesity-related health risk. American Journal of Clinical Nutrition 2004; 79: 379-384.

26. Alexander, C. M., Landsman, P. B., Teutsch, S. M., Haffner, SM. NCEP-defined metabolic syndrome, diabetes, and prevalence of coronary heart disease among NHANES III participants age 50 years and older. Diabetes 2003; 52: 1210-1214.

27. Alenezi, M. Y., M. Marcil. Is the Decreased High-Density Lipoprotein Cholesterol in the Metabolic Syndrome Due to Cellular Lipid Efflux Defect? J. Clin Endocrinol Metab 2004; 89(2): 761-764

28. Brasier AR, Resinos A III, Eledrisi MS. Vascular inflammation and the renin-angiotensin system. Arterioscler Thromb Vasc Biol 2002; 22: 1257-66

29. Knopp RH, Paramsothy P. Oxidized LDL and abdominal obesity: a key to understanding the metabolic syndrome. Am J Clin Nutr 2006; 83: 1-2.

30. Hansel B, Giral P, Nobecourt E, Chantepie S, Bruckert E, Chapman J. Metabolic syndrome is associated with elevated oxidative stress and dysfunctional dense highdensity lipoprotein particles displaying impaired antioxidative activity. J Clin Endocrinol Metab 2004; 89: 4963-71.
31. Furukawa, S., T. Fujita. Increased oxidative stress in obesity and its impact on metabolic syndrome. Journal of Clinical Investigation 2004; 114(12): 1752.

32. Holvoet, P., S. B. Kritchevsky. The Metabolic Syndrome, Circulating Oxidized LDL, and Risk of Myocardial Infarction in Well-Functioning Elderly People in the Health, Aging, and Body Composition Cohort. Diabetes 2004; 53(4): 1068-1073.

33. Veena S. Rao, Radhika K. Nagaraj, Sridhara Hebbagodi, Natesha B. Kadarinarasimhiah, and Vijay V. Kakkar. Association of Inflammatory and Oxidative StressMarkers with Metabolic Syndrome in Asian Indians in India. Cardiology Research and Practice 2011; 10: 4061-69.
Publish in International Archives of Medicine

International Archives of Medicine is an open access journal publishing articles encompassing all aspects of medical science and clinical practice. IAM is considered a megajournal with independent sections on all areas of medicine. IAM is a really international journal with authors and board members from all around the world. The journal is widely indexed and classified Q1 in category Medicine. 